\title{
Kapitalismus im Wachstumsdilemma. Die Verdrängung der ökologischen Krisen- dimension und ihre Folgen
}

\author{
KLAUS DÖRRE
}

Wenige Jahre nach dem globalen Finanzcrash ist das Gespenst der Rezession zurück. Selbst in „Gewinnerstaaten“ wie der Bundesrepublik machen sich die „Sturmvögel der Krise“ (Marx) bemerkbar. Einige südeuropäische Krisenländer haben das rezessive Tal erst gar nicht verlassen. Gegen die Stagnation scheint es nur ein Mittel zu geben - die Generierung von Wirtschaftswachstum. Bei der Wahl ihrer Mittel im grundlegenden Dissens, wissen sich Monetaristen und Keynesianer hin sichtlich der Suche nach Wachstumstreibern einig. Darf man diesen stillen Konsens attackieren? Meine Antwort ist ein klares Ja! Die Gesellschaften des globalen Nordens befinden sich in einem Wachstumsdilemma. Weil alle Energien auf eine ausschließlich wachstumsorientierte Überwindung der ökonomischen Stagnation gerichtet sind, geraten ökologische Nachhaltigkeitsziele zunehmend unter die Räder - dies mit fatalen Folgen vor allem für die Gesellschaften des globalen Südens.

\section{Modernes Wachstum}

Zur Begründung dieser These lohnt eine Beschäftigung mit dem Wachstumsbegriff. „Wir wünschen uns Wachstum, nicht Stillstand oder Schrumpfung. Und das ist menschlich", $\ominus^{\ominus}$ behauptet der liberale Ökonom Karl-Heinz Paqué. Eine solche Gleichsetzung von Wirtschaftswachstum und Entwicklung ist jedoch historisch falsch. Modernes Wachstum kann als eine von Preisbewegungen unabhängige Mehrung des Volks- und Pro-Kopf-Einkommens bezeichnet werden, die sich über alle konjunkturellen Schwankungen hinweg verstetigen lässt. Dergleichen gibt es erst seit der industriellen Revolution und dem Aufstieg des Industriekapitalismus. Einiges spricht dafür, dass der Akkumulator langsamer Fortschritte wirtschaftliche Leitsektoren und Regionen, die als Lokomotiven der Weltwirtschaft fungieren - das „Ob“ längerfristigen Wachstums bestimmt. Von dieser Bewegung müssen kurze Intervalle, Konjunkturzyklen, unterschieden werden, in deren Verlauf Anlage- und Unternehmensstrategien, aber auch wirtschaftspolitische Entscheidungen das „Wie“ des Wachstums beeinflussen.

\section{Ein systemischer Wachstumszwang}

Kapitalistischen Ökonomien ist ein struktureller Wachstumszwang inhärent. Ohne die erweiterte Reproduktion des eingesetzten Kapitals und die fortwährende Steigerung der Arbeitsproduktivität lässt sich kapitalistisches Gewinnstreben letztendlich nicht realisieren. Um Gesellschaften mit eingebautem Wachstumszwang ökonomisch und sozial zu stabilisieren, müssen sie sich permanent bewegen, ihre Volkswirtschaften müssen wach sen. Zugespitzt formuliert: Die dem Kapitalismus in all seinen Variationen innewohnende Dynamik treibt das System ,immer nur in ein Extrem - in die Expansion oder in den Zusammenbruch". ${ }^{\text {(2) }}$ Kapitalistische Dynamik ist daher nichts anderes als der fortwährende Versuch vor allem großer Unternehmen und der Staaten, Schranken der Akkumulation und damit auch Möglichkeitsgrenzen des Wachstums zumindest zeitweilig zu überwinden und räumlich zu verschieben, wobei jede dieser Grenzen immer wieder von einer anderen abgelöst werden kann. In diesem Sinne verkörpert modernes Wachstum eine „Bewegung von Relais zu Relais“, von Grenze zu Grenze, „ohne je zum Stillstand zu kommen“. ${ }^{3}$

Kapitalistische Dynamik bleibt auf die fortwährende Einverleibung von „,neuem Land“, präziser: von zuvor nicht kommodifizierten Territorien, sozialen Milieus und Arbeitskräften angewiesen. Wohlfahrtsstaaten haben diesen ökonomischen Wachstumszwang auch gesellschaftlich verallgemeinert und politisch institutionalisiert. Der Sozialstaat darf „die Stabilitätsanforderungen des kapitalistischen Wachstums“ nicht verletzen, weil privilegierte Eliten „korri- gierende Eingriffe in das Verteilungsmuster" nur dann akzeptieren, wenn „sie aus den Zuwächsen des Sozialprodukts bestritten werden " können und die Besitzstände dieser Gruppen nicht berühren. ${ }^{\circledR}$ Dementsprechend lässt sich die Restrukturierung der fortgeschrittenen Kapitalismen seit Mitte der 1970er Jahre als Abfolge von Versuchen verstehen, die längerfristige Wachstumsdynamik - zunächst mittels staatlicher Kreditierung, später über eine angebotsorientierte oder neoschumpeterianische Wirtschaftspolitik - wieder in Gang zu bringen. Die „große Kontrak tion“" von 2008/09 signalisiert das Scheitern dieser Versuche.

\section{Selektivität des Wachstums}

Dafür spricht zunächst die relative Entkopplung von Wirtschaftswachstum und sozialer Wohlfahrt, wie sie sich seit Längerem beobachten lässt. War schon gegen Ende der fordistischen Ära eine zunehmende Nord-Süd-Spaltung sichtbar geworden, so hat sich seither ein selektives Muster durchgesetzt, das auch innerhalb des globalen Südens polarisiert. Den dynamischen Wachstumsökonomien in den BRICS-Staaten ${ }^{\ominus}$

\footnotetext{
Paqué, K. H. (2010): Wachstum! Die Zukunft des globalen Kapitalismus, München, S. 251.

(2) Jackson, T. (2011): Wohlstand ohne Wachstum, München, S. 80.

3 Braudel, F. (1986): Sozialgeschichte des 15.-18 Jahrhunderts. Aufbruch zur Weltwirtschaft, München, S. 662

4 Habermas, J. (1987): Theorie des kommunikativen Handelns, Bd. 2, Frankfurt a. M., S. 511.

(5) Die Abkürzung BRICS steht für die Anfangsbuchstaben der fünf Staaten: Brasilien, Russland, Indien, China und Südafrika.
} 
und besonders in Ostasien stehen Länder gegenüber, in denen die Rezession zum Dauerzustand geworden ist. Bereits zur Jahrtausendwende war das Pro-Kopf-Bruttoinlandsprodukt in 54 Staaten mit immerhin $12 \%$ der Weltbevölkerung seit mehr als einem Jahrzehnt rückläufig. Zwar gilt nach wie vor, dass Wachstumsstockungen eine Zunahme von Arbeitslosigkeit, Armut und Prekarität bedeuten; als das Wachstum des Bruttoinlandsprodukts (BIP) 2008/09 einbrach, gingen allein in China ca. 20 Mio. Jobs verloren. Doch das Anziehen der Weltkonjunktur führte global weder zu sinkenden Arbeitslosenquoten noch zu einer Korrektur des Polarisierungstrends bei Einkommen und Vermögen. Obwohl die Weltwirtschaft 2010 um $5 \%$ und 2011 noch einmal durchschnittlich um $4 \%$ gewachsen ist, waren 2011 noch immer 197 Mio. und damit 27 Mio. Menschen mehr arbeitslos als vor der Krise; 900 Mio. Menschen lebten laut der Internationalen Arbeitsorganisation (ILO) unter der absoluten Armutsschwelle von 2 US-Dollar pro Tag.

Die soziale Selektivität des Wachstums macht sich aber auch innerhalb der fortgeschrittenen Kapitalismen bemerkbar. So beruht das ,deutsche Beschäftigungswunder" wesentlich auf einer Expansion unsicherer Beschäftigung. Im Entstehen ist eine prekäre Vollerwerbsgesellschaft, in welcher die einkommensschwächsten Gruppen nicht oder kaum an Produktivitätsfortschritten partizipieren. DAX-Manager, die sich vor 20 Jahren noch mit dem Vierzehnfachen eines durchschnittlichen Facharbeitergehalts begnügten, verdienen heute durchschnittlich das Zweiundfünfzigfache. Demgegenüber arbeiten inzwischen mehr als $23 \%$ der abhängig Beschäftigten im Niedriglohnsektor, davon gut 3,4 Mio. mit einem Bruttolohn von weniger als $7 €$ pro Stunde.

\section{Ökologische Grenzen}

Das alles sei kein Problem des Wirtschaftswachstums, sondern seiner gerechten Verteilung - so könnte ein Einwand lauten. Doch solche Argumente ignorieren die ökologischen Wachstumsgrenzen. Das Jahr 2009 lieferte dafür Anschauungsunterricht. Nicht etwa höhere Ressourceneffizienz oder beschleunigtes Umsteigen auf erneuerbare Energien, sondern ökonomisches Minuswachstum sorgte für einen Rückgang klimaschädlicher Emissionen. Als die Konjunktur 2010 anzog, war das Rekordniveau der Emissionen aus 2008 jedoch rasch wieder erreicht. Der globale $\mathrm{CO}_{2}$-Ausstoß übertrifft inzwischen noch die pessimistischsten Prognosen des UN-Klimarates. Die jüngste Studie des Club of Rome, die einen „Peak 2030“ für die Klimaintensität, einen Anteil erneuerbarer Energien von $40 \%$ und eine Stagnation der Weltbevölkerung bei 8,1 Mrd. Menschen (2040) annimmt, hält eine Erderwärmung von vier bis fünf Grad bis zum Jahrhundertende für wahrscheinlich. ${ }^{\odot}$ Folgt man dem OECD-Umweltausblick, der bis 2050 eine Vervierfachung der Weltwirtschaft, 9 Mrd. Menschen, nur $15 \%$ erneuerbare Energieträger, einen um 80 \% erhöhten Energiebedarf und 70 \% mehr $\mathrm{CO}_{2}$-Emissionen prognostiziert, so ist das eher noch ein optimistisches Szenario.

Treibhausemissionen und Klimawandel stellen jedoch nur eine Dimension ökologischer Krisenherde dar. Nicht minder gravierend ist die Vernutzung endlicher natürlicher Ressourcen und fossiler Energieträger. An den endlichen Ressourcen gemessen, lebt die Welt schon seit dem Ende der 1970er Jahre über ihre Verhältnisse. Der ökologische Fußabdruck, der den Ressourcenverbrauch im Verhältnis zur ökologischen Tragfähigkeit des Planeten misst, ist dafür ein wichtiger Indikator. Schon vor der Jahrtausendwende lag der menschliche Ressourcenverbrauch etwa 20 \% über der ökologischen Tragfähigkeit. Und auch hier zeigt sich eine Parallelität zu den Emissionen. In den Krisenjahren zwischen 1980 - 1983 näherte sich der Ressourcenverbrauch zuletzt der Tragfähigkeitsgrenze an. Seither ist er in einem Maße gestiegen, der die „Möglichkeitsgrenzen“ (Braudel) längerfristigen Wirtschaftswachstums zumindest in den fortgeschrittenen Kapitalismen immer näher rücken lässt. ${ }^{0}$ Diese Tendenz ist umso brisanter, als sie bei Teilen der ökonomischen und politischen Eliten noch immer die Bereitschaft fördert, hochrisikoträchtige Technologien wie Kernkraftwerke zum Einsatz zu bringen.

Wegen der Kumulation solcher Risiken macht es Sinn, die gegenwärtige Konstellation als ökonomisch-ökologische Doppelkrise zu bezeichnen. Weder ökologische noch ökonomische Krisen lassen sich auf die eine Ursache zurückführen. Der Begriff Doppelkrise bezeichnet lediglich eine räumliche und zeitliche Synchronisation höchst unterschiedlicher Krisenherde und -ursachen. Das historisch Neue der gegenwärtigen Krisenkonstellation besteht jedoch darin, dass das Wachstumsdilemma fortgeschrittener Kapitalismen offen zutage tritt. Das seit Jahrzehnten fraglos eingesetzte Mittel zur Überwindung ökonomischer Krisen, die Generierung von materiellem Wachstum, bewirkt in der Gegenwart fast zwangsläufig eine Zuspitzung und Verschärfung ökologischer Krisen. Aus diesem Grund befinden sich die Gesellschaften des globalen Nordens in einer historischen Entscheidungssituation: Entweder es gelingt ihnen, Wirtschaftswachstum ökologisch und sozial nachhaltig zu gestalten, oder sie sind gezwungen, ihre Entwicklung und das heißt auch: ihre wohlfahrtsstaatlichen und sozialen Institutionen - dauerhaft vom Zwang zu permanentem Wachstum zu entkoppeln.

\section{Wege aus der Krise}

Wichtig ist, dass diese historische Entscheidungssituation nicht im Sinne einer apokalyptischen Prophezeiung interpretiert wird. Alle bekannten Prognosen über den Klimawandel und die Grenzen des Wachstums basieren auf hochkomplexen Computersimulationen, deren Ergebnisse hochgradig von den eingespeisten Prämissen abhängen. Auch weil sie auf defizitären Informationen beruhen, dürfen sie nicht im Sinne exakter $\mathrm{Zu}$ kunftsbeschreibungen missverstanden werden. Verdichtet man, wie einst Rudolf Bahro, die pessimistischsten Szenarien zur Negativutopie einer bevorstehenden Apokalypse, spielt dies ungewollt den Wachstumsfetischisten in die Hände. Jeder noch so begrenzte Fortschritt beim Klima oder den Ressourcen - die Lachse im Rhein, Dächer mit Sonnenkollektoren oder der gelegentlich wieder blaue Himmel über der Ruhr - kann dann als praktische Widerlegung hermetischer Untergangszenarien erscheinen. Die Akzeptanz der Untergangsszenarien liefe hingegen auf die Erzeugung einer fatalistischen Opfermentalität hinaus.

Gegen apokalyptische Visionen betont das Konzept der ökonomisch-ökologischen Doppelkrise die politische Beeinflussbarkeit nicht nur der Ökonomie, sondern auch der gesellschaftlichen Naturverhältnisse. Gerade die Erschütterungen des kapitalistischen Weltsystems, die der Zangengriff von wirtschaftlichen und ökologischen Verwerfungen auslöst, erzeugt Spielräume für radikale Veränderungen. Doch wie soll interveniert werden? Noch weit davon entfernt, die Leitlinien politischen Handelns zu bestimmen, sind es diverse Varianten eines Green New Deal, die sich als alternative Option anbieten. In der konkreten Ausgestaltung sehr unterschiedlich, beanspruchen sie, zwei Fliegen mit einer Klappe

6 Randers, J. (2012): 2052: A Global Forecast For the Next Forty Years: Chelsea Green Publishing.

$(7$ Meadows, D./Randers, J./Meadows, D. (2012): Grenzen des Wachstums. Das 30-Jahre-Update. Signal zum Kurswechsel, Stuttgart, S. XVII. 
zu schlagen. Das Feld ökologischer Investitionen und grüner Sprunginnovationen wird als potenzieller Akkumulator entdeckt, der sowohl längerfristiges Wachstum als auch dessen Dekarbonisierung und Dematerialisierung ermöglichen soll.

Gegenüber der konservativ gefärbten Wachstumskritik eines Meinhard Miegel, die bei fortbestehenden Ungleichheiten Wohlfahrtsverzicht für die breite Masse empfiehlt, stellen solche Konzepte einen Fortschritt dar. Reflektieren sie doch die Zentralität sozialer Nachhaltigkeit für den dringend benötigten ökologischen Umbau. In Gesellschaften mit ausgeprägten Ungleichheiten und Klassenunterschieden wird auch der Kampf um positionale Güter besonders erbittert geführt. Die Verschärfung horizontaler Ungleichheiten heizt deshalb den Statuskonsum an. Schritte in Richtung ökologischer Nachhaltigkeit sind daher in egalitären Gesellschaften leichter möglich. Die besseren Varianten eines Green New Deal könnten die Spielräume für Umverteilungspolitiken zugunsten der Lohneinkommen zeitweilig sicher vergrößern - und dies bei gleichzeitiger Verbesserung der Ressourceneffizienz und -effektivität. Eine Garantie für eine Umverteilung zugunsten der Prekarisierten und der vom Wachstum abgehängten Länder beinhalten sie nicht. Auch deshalb stellen sie längerfristig keinen Ausweg aus dem Wachstumsdilemma dar. Gegenwärtig konsumiert ein Viertel der Weltbevölkerung vorwiegend des globalen Nordens drei Viertel der Ressourcen und erzeugt drei Viertel des Abfalls und der Emissionen. Diese Problematik lässt sich allein mittels Dematerialisierung und Dekarbonisierung des Wachstums nicht entschärfen. Reboundeffekte, erzeugt durch größeren Output und steigenden Konsum, stellen die Erfolge solcher Strategien früher oder später wieder in frage. Das qualitative Wachstum bliebe so „wenig nachhaltig wie bisher“. ${ }^{8}$

\section{Demokratische Transformation}

Sozialökologische Investitions- und Innovationsprogramme, etwa ein von den Gewerkschaften geforderter Marshallplan für die südeuropäischen Krisenländer, sind deshalb nicht falsch. Im Gegenteil, politisch realisiert, würden sie ökologisch zumindest Zeitgewinn bedeuten. Für eine Nachhaltigkeitsrevolution sind sie als Übergangstrategien sinnvoll, aber eben nicht ausreichend. Offenkundig steht eine Transformation bevor, die das moderne Wachstum als ökonomischen
Selbstzweck infrage stellt. Nur wenn die gesellschaftliche Entwicklung im Norden vom Zwang zu stetig steigendem Wachstum entkoppelt wird, hat der globale Süden überhaupt noch eine Entwicklungschance. Ein Übergang zu Postwachstumsgesellschaften scheint derzeit utopisch, weil dies den Wesenskern kapitalistischer Vergesellschaftung berührt. Das könnte sich jedoch rasch ändern, wenn sich die ökologischen Verwerfungen in den Zentren auch ökonomisch und sozial bemerkbar machen. Wenn Energie und Elektrizität nicht mehr immer und - preisbedingt - auch nicht mehr für alle in gleichem Maße zu Verfügung stehen, könnte sich auch hierzulande rasch ein Trend bemerkbar machen, den die jüngste Club-of-Rome-Studie schon jetzt für wahrscheinlich hält: Die größten Fortschritte bei den erneuerbaren Energien trauen die Autoren China zu - nicht trotz, sondern wegen fehlender Demokratie!

Wer sich autoritären Anwandlungen nicht ohnmächtig ausliefern will, muss daher schon jetzt mit der Suche nach demokratischen Alternativen beginnen. Dafür gibt es Anknüpfungspunkte. Kapitalismus existiert niemals in Rein form. Seine Wirtschaft ist von Sektoren abhängig, die weder nach Wachstumsimperativen funktionieren noch dem Profitmotiv gehorchen. So ist die Inwertsetzung von zunehmend flexibler Produktionsarbeit auf ein immer größeres Volumen von ernährenden, erziehenden, bildenden, pflegenden und sorgenden Tätigkeiten angewiesen. Diese Tätigkeiten, häufig Domänen von Frauen, werden überwiegend schlecht bezahlt oder gar gratis genutzt. Sie sind Sphäre von Überausbeutung und Diskriminierung. Für solche Dienstleistungstätigkeiten gilt, dass die Wegrationalisierung menschlicher Arbeitskraft zwangsläufig zulasten der Dienstleitungsqualität geht. Wenn überhaupt, so sind es jedoch diese relativ rationalisierungsresistenten Sektoren, die in den fortgeschrittenen Ländern langsam, aber stetig wachsen können. Sie zu fördern, bedeutete den allmählichen Bruch mit einem ressourcen-, energie- und schadstoffintensiven Wachstumstyp, der Arbeitskraft durch Technik, durch totes Kapital ersetzt. Die Aufwertung und bessere Bezahlung

(8) Jackson, T. (2011), a.a.O. (Fußnote 2), S. 129.

(9) Wright, E. O. (2012): Transformation des Kapitalismus, in: Dörre, K./Sauer, D./Wittke, V.(Hrsg.): Kapitalismustheorie und Arbeit. Neue Ansätze soziologischer Kritik, Frankfurt a. M./New York, S. $462-488$. eines Teils dieser Tätigkeiten würde einen Paradigmenwechsel in der Finanz- und Steuerpolitik voraussetzen. Es ginge um die Finanzierung qualifizierter Humandienstleistungen, um die Bereitstellung öffentlicher Güter in einem Sektor, der genossenschaftlich organisiert sein könnte. Ein solcher Sektor würde ergänzend innovative Verzahnungen von Öffentlichem und Privatem benötigen, denn nicht alle Pflegeleistungen können öffentlich erbracht werden. Er hätte die Demokratisierung von Dienstleistungsarbeit durch Mitbestimmung von Produzenten und Klienten zu fördern. Geschlechtergerechte Arbeitszeitverkürzungen und bezahlte Zeit für Arbeit an der Demokratie würden ebenfalls zu einem Programm gehören, das sinnvolle Arbeit an die Stelle des Steigerungsprinzips eines „Immer mehr und nie genug!“ setzte.

Eine solche Transformation käme nicht ohne die öffentliche Kontrolle gesellschaftlicher Schlüsselsektoren (Energie, Finanzen) aus, weil ökologische Investitionen sich häufig nur auf lange Sicht amortisieren. Sie hätte die großen marktbeherrschenden Unternehmen zu dem zu machen, was sie implizit bereits sind - zu öffentlichen Institutionen, deren Aktivitäten an einen demokratischen Kollektivwillen gebunden sind. Dies wäre eine Transformationsperspektive, die in einem allgemeineren Sinne die Stärkung gesellschaftlicher gegenüber ökonomischer und staatlicher Macht und damit die Erweiterung von Demokratie bedeuten würde. ${ }^{\ominus}$ Politische Akteure, auch Gewerkschaften, setzten ihr Zukunftsfähigkeit aufs Spiel, wollten sie sich der Diskussion über eine solch radikale gesellschaftliche Transformation verweigern. Die Aussichten auf einen demokratischen Wandel hin zu sozialer und ökologischer Nachhaltigkeit mögen gegenwärtig nicht berauschend sein. Sie endlich in Angriff zu nehmen, ist jedoch allemal besser, als sich in blauäugiges Wunschdenken oder zynische Opfermentalität zu flüchten.

\section{AUTOR}

KLAUS DÖRRE, Prof. Dr., lehrt Soziologie an der Universität Jena und ist einer der Sprecher des DFG-Forschungskollegs „Postwachstumsgesellschaften“. Arbeitsschwerpunkte: Kapitalismustheorie/Finanzmarktkapitalismus, flexible und prekäre Beschäftigung, Arbeitsbeziehungen, Green New Deal.

klaus.doerre@uni-jena.de 